\title{
TIME CORRELATION FUNCTIONS FOR THE ONE-DIMENSIONAL LORENTZ GAS
}

\author{
R M MAZO* and Henk VAN BEIJEREN \\ Instttut fur Theoretische Physik $A$ and B, RWTH Aachen SommerfeldstraBe, D-5100 Aachen, \\ Germany
}

Recelved 11 October 1982

\begin{abstract}
The velocity autocorrelation function and related quantities are investigated for the onedimensional deterministic Lorentz gas, consisting of randomly distributed fixed scatterers and light particles moving back and forth between two of these at a constant given speed An expansion for the velocity autocorrelation function given by Grassberger, which is useful for short times, is reconstructed The long tıme behavior is investigated by Fourier transform techniques For large time $t$ the velocity autocorrelation function decays as $\exp \left(-c t^{1 / 2}\right)$ and in addition oscillates with a period increasing as $t^{1 / 2} \mathrm{~A}$ velocity average over a Maxwellian changes this long tıme behavior to $\exp \left(-c^{\prime} t^{2 / 3}\right)$, while the oscillations are removed The Green's function is also investigated Its spatial and temporal Fourier transform, the incoherent scattering function, exhibits strongly non-Lorentzian behavior
\end{abstract}

\section{Introduction}

The asymptotic long time behavior of correlation functions connected with transport phenomena, has been a subject of appreciable interest for several years One of the best-known examples is the velocity autocorrelation function, obtained by multiplying the velocity of a tagged particle at an initial instant of time by the velocity of the same particle at a given time $t$ later and subjecting this product to an equilibrium average. The time integral from zero to infinity of this velocity autocorrelation function, if it exists, equals the coefficient of self-diffusion or tracer diffusion ${ }^{1}$ ).

Contrarious to previous expectations the velocity autocorrelation function of a tagged particle in a fluid exhibits a long time behavior proportional to $t^{-d / 2}$ in $d$ dimensions ${ }^{2}$ ) (at least for $d>2$, for the subtle corrections occurring if $d=2$, see ref 3 and 4 ) as a result of conservation of particles and momentum For diffusive systems in which momentum is not conserved but particles are, the asymptotic long time behavior reduces to a $t^{-(d / 2+1)}$ law $\mathrm{A}$ well-known example of such a system is the Lorentz gas $^{5,6}$ ), in which mutually noninteracting point particles move at constant speed among fixed hard spherical

\footnotetext{
* Permanent address Dept of Chemistry, University of Oregon, Eugene, Or 97403, USA
} 
scatterers, making specular collisions with the latter on collisions. For low densities of scatterers Ernst and Weyland ${ }^{7}$ ) derived the above mentioned $t^{-(d / 2+1)}$-law for this system, subsequently this was confirmed in computer simulations ${ }^{8-10}$.

A direct consequence of a $t^{-\alpha}$ long time behavior of the velocity autocorrelation function is the occurrence of a term proportional to $t^{2-\alpha}$ (or $\log t$ if $\alpha=2$ ) in the mean square displacement of the tagged particle ${ }^{11}$ ). Hence, for $\alpha \leqslant 2$ the mean square displacement increases indefinitely with time. There are systems, however, in which this cannot occur For example, if in the Lorentz gas the scatterers are allowed to overlap each other, there exists a critical density of scatterers, called the percolation density, above which all moving particles are trapped within finite volumes bounded by scatterers ${ }^{10,12-14}$ ). At scatterer densities above this percolation density the mean square displacement of a tagged particle, determined by averaging over all free volumes in the system, is strictly bounded for all times since large volumes have a probability of occurrence that decreases rapidly as their diameter increases From this point of view it is remarkable that Alley and Alder ${ }^{10}$ ) found a $t^{-\alpha}$ long time behavior of the velocity autocorrelation function, with $\alpha<2$, for a two-dimensional Lorentz gas with overlapping scatterers, at densities far beyond the percolation density. One cannot but conclude that this result represents an intermediate time behavior and not an asymptotic law. Theoretical explanations for such intermediate time behavior are lacking.

An extremely simple system with similar features is the one-dimensional Lorentz gas. In this system point scatterers are Poisson distributed on a line and the light particles simply keep running back and forth between two neighboring scatterers. To get beyond this simple behavior Grassberger ${ }^{15}$ ) recently proposed a stochastic version of the one-dimensional Lorentz gas, in which the moving particles at an encounter with a scatterer have probability $p$ of being reflected and probability $1-p$ of being transmitted with unchanged velocity It could be shown ${ }^{15,16}$ ) that for this stochastic model the velocity autocorrelation function of a moving particle exhibits the $t^{-3 / 2}$ long time tail, to be expected for a one-dimensional diffusive Lorentz gas In the limit $p \rightarrow 1$ the stochastic Lorentz gas reduces to the deterministıc Lorentz gas described above, which provides some interest to the properties of the latter.

Grassberger investigated by Monte-Carlo simulations the velocity autocorrelation function of a particle moving at a given constant speed, for a number of values of the reflection parameter $p$ For $p=0.5$, and more strongly for $p=075$, he found fairly persistent oscillations with a period that seemed to increase with time and which he attributed to the $p=1$ limit making itself felt already Similar results were obtained, but not published, by Erpenbeck ${ }^{17}$ ) and by Dumcke ${ }^{18}$ ) Grassberger calculated the velocity autocorrelation function in 
the deterministic limit $p=1$ analytically in the form of a series expansion By numerical summation he found that indeed the velocity autocorrelation function keeps oscillating over an appreciable time range. However, the questions whether these oscillations persist for all times and how do their period and amplitude change with time, cannot easily be answered on the basis of this series expansion. It is our goal in this paper to investigate the asymptotic behavior of the velocity autocorrelation function and some closely related functions, of the deterministic one-dimensional Lorentz gas. Although basically simple, the mathematics of this problem is amusing and, in view of the above considerations, the results to our feeling are of some, albeit perhaps restricted, interest. The main results are as follows: the velocity autocorrelation function keeps oscillating indefinitely, the amplitude decreases as $\exp -\left(\omega_{0} t\right)^{1 / 2}$, with $\omega_{0}$ the average angular frequency of the light particle motion, and the period of the oscillation of the velocity autocorrelation function increases as $4 \pi\left(t / \omega_{0}\right)^{1 / 2}$ with time. One may hope that these results will also give a qualitative indication of the long time behavior of the velocity autocorrelation function in higher dimensional Lorentz gases at above-percolation densities Then the next step would be to consider the motion of localized but locally mobile particles in glasses or other disordered dense systems. In that case, however, one cannot assume, as in the model calculations, that all moving particles have the same speed; instead a Maxwell distribution is called for. We have applied such a distribution to our velocity autocorrelation function and find it decays even faster than for the case of a single speed.

In the remainder of this paper we will consider the velocity autocorrelation function, its Fourier transform or power spectrum, the Green's function $P(x$, $t)$ describing the probability to find a particle starting off at the origin at time $t=0$, at position $x$ at time $t$, and its Fourier and Laplace transform, the incoherent scattering function.

We will assume the intervals between neighboring scatterers to be Poisson distributed with an average length $L$. For the light particles, moving at velocity $\pm v$, we will consider two types of distributions: first the equilibrium distribution, in which the probability to find a light particle in a given interval is proportional to the length of the interval, and secondly a "quenched distribution", obtained by first freezing in the scatterers at a high density, leading to an exponential distribution of interval lengths with average spacing $L$, and next injecting light particles of a diameter $a \gg L$. In this case the probability of finding intervals that can hold two light particles is negligible and the light particles may be assumed to be distributed uniformly over the few intervals large enough to hold one. The free length (the distance over which the light particle can move) of an interval of length $x$ equals $l=x-a$, 
and the distribution for $l$ is again an exponential distribution of the form $L^{-1}(\exp (-l / L))$.

In the next chapters we consider the equilibrium distribution first, the changes resulting from passing to the quenched average are presented separately.

\section{The velocity autocorrelation function}

\subsection{Grassberger's series expansion}

For a light particle moving at constant speed $v$ on an interval of fixed length $l$, the velocity autocorrelation function is obtained by averaging over all initial positions on the interval and the two possible initial velocities, with the result

$$
\begin{aligned}
\langle v(0) v(t)\rangle_{\tau}= & v^{2} \sum_{n=-\infty}^{\infty}\left[\left\{\Theta(t-n \tau)-\Theta\left(t-\left(n+\frac{1}{2}\right) \tau\right)\right\}\left(1-\frac{4(t-n \tau)}{\tau}\right)\right. \\
& \left.+\left\{\Theta\left(t-\left(n-\frac{1}{2}\right) \tau\right)-\Theta(t-n \tau)\right\}\left(1-\frac{4(n \tau-t)}{\tau}\right)\right] .
\end{aligned}
$$

Here we introduced the period $\tau=2 l / v$ and the unit step function $\Theta(x)$, which equals unity for $x \geqslant 0$ and vanishes for $x<0$ The brackets indicate an averaging and the subscript $\tau$ is used of this averaging is an equilibrium average over an interval with period $\tau$. The time argument $t$ may be positive or negative. By a simple algebraic rearrangement the above equation may be rewritten as

$$
\begin{aligned}
\langle v(0) v(t)\rangle_{\tau}= & v^{2} \sum_{n=-\infty}^{\infty}\left[\left\{\Theta(t-n \tau)-\Theta\left(t-\left(n+\frac{1}{2}\right) \tau\right)\right\}\left(1-\frac{2(t-n \tau)}{\tau}\right)\right. \\
& +\left\{\Theta\left(t-\left(n-\frac{1}{2}\right) \tau\right)-\Theta(t-n \tau)\right\}\left(1-\frac{2(n \tau-t)}{\tau}\right) \\
& -\left\{\Theta\left(t-\left(n+\frac{1}{2}\right) \tau\right)-\Theta(t-(n+1) \tau)\right\}\left(1-\frac{2\left(t-\left(n+\frac{1}{2}\right) \tau\right)}{\tau}\right) \\
& -\left\{\Theta(t-n \tau)-\Theta\left(t-\left(n+\frac{1}{2}\right) \tau\right)\right\}\left(1-\frac{2\left(\left(n+\frac{1}{2}\right) \tau-t\right)}{\tau}\right) \\
\equiv & \sum_{n=-\infty}^{\infty} f_{n}(t, \tau)
\end{aligned}
$$

Note that the series in both eqs. (1) and (2) are absolutely convergent. Next this purely periodic expression must be averaged over the distribution for the period $\tau$. In equilibrium, where the interval lengths are Poisson distributed and, in a fixed configuration, the probability to find a light particle on an 
interval of length $l$ is proportional to $l$, the distribution for $\tau$ assumes the form

$$
P_{\mathrm{eq}}(\tau)=\frac{\tau}{T^{2}} \exp (-\tau / T)
$$

with $T=2 L / v$ and $L$ is the average length of an interval between neighboring scatterers Combining (2) and (3) one finds for the velocity autocorrelation function

$$
\begin{aligned}
\langle v(0) v(t)\rangle_{\mathrm{eq}}= & \int_{0}^{\infty} \mathrm{d} \tau\langle v(0) v(\tau)\rangle_{r} P_{\mathrm{eq}}(\tau) \\
= & \int_{0}^{\infty} \mathrm{d} \tau P_{\mathrm{eq}}(\tau) \sum_{n=-\infty}^{\infty} f_{n}(t, \tau) \\
= & v^{2}[3 \exp (-2|t| / T)-2 \exp (-|t| / T) \\
& +\sum_{n=2}^{\infty}(-1)^{n}\left\{(n-1) \exp \frac{-2|t|}{(n-1) T}-2 n \exp \frac{-2|t|}{n T}\right. \\
& \left.\left.+(n+1) \exp \frac{-2|t|}{(n+1) T}\right\}\right],
\end{aligned}
$$

which is Grassberger's result ${ }^{15}$ ) The sum in (4) is absolutely convergent, as follows from

$$
\int_{0}^{\infty} \mathrm{d} \tau P(\tau) \sum_{n}\left|f_{n}(t, \tau)\right|=v^{2} \int_{0}^{\infty} \mathrm{d} \tau P(\tau)=v^{2}
$$

If one would rewrite (4) into the apparently simpler form

$$
\langle v(0) v(t)\rangle_{\mathrm{eq}}=\sum_{n=1}^{\infty}(-1)^{n+1} 4 n \exp \frac{-2|t|}{n},
$$

the absolute convergence would have been lost. For not too long times the convergence of the series in (4) is farly rapid Grassberger used it to evaluate the velocity autocorrelation function by numerical summation up till $t=5 \mathrm{~T}$. The form of eq. (4) is not suitable, however, for extracting the long time behavior in a simple way

\subsection{Fourier series representation and long time behavior}

Since the velocity autocorrelation function for a fixed interval length is a periodic function, as made explicit in eqs. (1) and (2), it is a natural throught 
to look for its Fourier series representation. This is well known, and easily constructed, with the result

$$
\langle v(0) v(t)\rangle_{\tau}=\sum_{n=-\infty}^{\infty} \frac{4 v^{2}}{[(2 n+1) \pi]^{2}} \exp \{(2 n+1) 2 \pi \mathrm{i} t / \tau\}
$$

Notice that this sum is absolutely and uniformly convergent again Averaging (6) with $P_{\text {eq }}(\tau)$, one obtains

$$
\begin{aligned}
\langle v(0) v(t)\rangle_{\mathrm{eq}} & =\int_{0}^{\infty} \mathrm{d} \tau \frac{\tau}{T^{2}} \mathrm{e}^{-\tau / T} \sum_{n=-\infty}^{\infty} \frac{4 v^{2}}{[(2 n+1) \pi]^{2}} \exp \{(2 n+1) 2 \pi \mathrm{i} t / \tau\} \\
& =\sum_{n=-\infty}^{\infty} \frac{16 v^{2} \mathrm{it}}{(2 n+1) \pi T} K_{2}\left\{(1+\mathrm{i}) \sqrt{(2 n+1) 2 \omega_{0} t}\right\}
\end{aligned}
$$

where $K_{2}$ is a Bessel function of imaginary argument, $\omega_{0}=2 \pi / T$ and the integrals over $\tau$ were found in Gradshteyn and Ryzhik ${ }^{19}$ ) In the same source one finds for the asymptotic long time behavior of $\mathrm{K}_{v}{ }^{20}$ ),

$$
\begin{aligned}
& \operatorname{Re}\left(K_{\nu}\left\{(1+\mathrm{i}) \sqrt{(2 n+1) 2 \omega_{0} t}\right\}\right) \simeq \frac{1}{2}\left(\frac{\pi}{2}\right)^{1 / 2}\left\{\frac{T}{(2 n+1) t}\right\}^{1 / 4} \\
& \quad \times \cos \left(\frac{1}{8} \pi+\left[(2 n+1) 2 \omega_{0} t\right]^{1 / 2}\right) \exp -\left[(2 n+1) 2 \omega_{0} t\right]^{1 / 2},
\end{aligned}
$$

where $\operatorname{Re}$ indicates the real part The result does not depend on the index $\nu$.

For large $t$ the term with $n=0$ (corresponding to the terms with $n=0,-1$ in (7)) dominates and the magnitude of the velocity autocorrelation function is seen to decay as $t^{3 / 4} \exp -\left(2 \omega_{0} t\right)^{1 / 2}$ In addition it keeps oscillating, with a period that increases as $(4 \pi t T)^{1 / 2}$ with time.

\section{Fourier transform of the velocity autocorrelation function}

Another quantity of interest is the Fourier transform, or power spectrum, of the velocity autocorrelation function. It can be interpreted as a frequency dependent diffusion coefficient or conductivity

$$
D(\omega)=\frac{1}{2} \int_{-\infty}^{\infty} \mathrm{d} t\langle v(0) v(t)\rangle \mathrm{e}^{-\imath \omega t} .
$$

For the calculation of this quantity different routes are available. If one wants to start from (4) he needs definitions and a few properties of the $\beta$-function, 
which is closely related to the $\Gamma$-function, namely ${ }^{21}$ )

$$
\begin{aligned}
& \beta(z)=\sum_{n=0}^{\infty} \frac{(-1)^{n}}{n+z}, \\
& \beta(z)-\beta(-z)-\frac{1}{z}=\pi / \sin (\pi z) .
\end{aligned}
$$

Inserting (4) into (9) one obtains

$$
\begin{aligned}
D(\omega)= & \frac{\pi v^{2}}{2}\left[\frac{3}{\omega_{0}+\mathrm{i} \pi \omega}+\frac{3}{\omega_{0}-\mathrm{i} \pi \omega}-\frac{4}{\omega_{0}+2 \mathrm{i} \pi \omega}-\frac{4}{\omega_{0}-2 \mathrm{i} \pi \omega}\right. \\
& +\sum_{n=2}^{\infty}(-1)^{n}\left\{\frac{(n-1)^{2}}{\omega_{0}+(n-1) \mathrm{i} \pi \omega}+\frac{(n-1)^{2}}{\omega_{0}-(n-1) \mathrm{i} \pi \omega}-\frac{2 n^{2}}{\omega_{0}+n \mathrm{i} \pi \omega}\right. \\
& \left.-\frac{2 n^{2}}{\omega_{0}-n \mathrm{i} \pi \omega}+\frac{(n+1)^{2}}{\omega_{0}+(n+1) \mathrm{i} \pi \omega}+\frac{(n+1)^{2}}{\omega_{0}-(n+1) \mathrm{i} \pi \omega}\right\} \\
= & \frac{2 v^{2} \omega_{0}^{2}}{\mathrm{i} \pi^{2} \omega^{3}}\left[\frac{\mathrm{i} \pi \omega}{\omega_{0}}+\sum_{n=1}^{\infty}(-1)^{n}\left\{\frac{1}{n+\omega_{0} / \mathrm{i} \pi \omega}-\frac{1}{n-\omega_{0} / \mathrm{i} \pi \omega}\right\}\right] \\
= & \frac{2 v^{2} \omega_{0}^{2}}{\mathrm{i} \pi^{2} \omega^{3}}\left[\beta\left(\frac{\omega_{0}}{\mathrm{i} \pi \omega}\right)-\beta\left(\frac{-\omega_{0}}{\mathrm{i} \pi \omega}\right)-\frac{\mathrm{i} \pi \omega}{\omega_{0}}\right] \\
= & \frac{2 v^{2} \omega_{0}^{2}}{\pi \omega^{3} \sinh \left(\omega_{0} / \omega\right)} .
\end{aligned}
$$

It is of interest to investigate the analytic structure of $D(\omega)$ in the complex $\omega$ plane. From the first line of (12) it is seen that the singularities of this function consist of an infinite series of simple poles, all located on the imaginary axis and with an accumulation point at the origin. Hence one may exclude exponential decay of the velocity autocorrelation function, faster than $\mathrm{e}^{-c t}$, with $c$ some positive constant, since in that case all poles would be located outside the strip $|\operatorname{Im}(\omega)|<c$ On the other hand one does not expect a power law decay either, because that would give rise to a branchcut along the imaginary axis. Indeed (12) suffices to show, without having to invoke the explicit result (7), that the velocity autocorrelation function decays faster than any inverse power of $t$. This is worked out in an appendix.

The more mundane way to derive (12) starts from (6), with the result

$$
\begin{aligned}
D(\omega) & =\frac{1}{2} \int_{0}^{\infty} \mathrm{d} \tau \frac{\tau}{T^{2}} \mathrm{e}^{-\tau / T} 2 \pi \sum_{n=-\infty}^{\infty} \frac{4 v^{2}}{((2 n+1) \pi)^{2}} \delta\left(\omega-\frac{2 \pi(2 n+1)}{\tau}\right) \\
& =\frac{4 v^{2} \omega_{0}^{2}}{\pi|\omega|^{3}} \sum_{n=0}^{\infty} \exp -\frac{(2 n+1) 2 \pi}{|\omega| T} \\
& =\frac{2 v^{2} \omega_{0}^{2}}{\pi \omega^{3} \sinh \left(\omega_{0} / \omega\right)} .
\end{aligned}
$$


The Fourier transform of $\langle x(0) x(t)\rangle$ can be easily obtained from $D(\omega)$ by multiplying by $2 \omega^{-2}$. This function is of interest because it would give the spectrum due to the translational motion of the impurity particle, were it charged and thus spectroscopically active. Notice that the spectrum is very non-Lorentzian.

\section{Series in powers of time}

To complete this section we give the representation of the velocity autocorrelation function as a power series in $t$. This may be obtained by expanding the exponentials in (4) and recollecting powers of $t$, with the result

$$
\begin{aligned}
\langle v(0) v(t)\rangle_{\mathrm{eq}}= & v^{2}\left[1-2 \nu t+2(\nu t)^{2} \log 2\right. \\
& \left.+4 \sum_{m=3}^{\infty} \frac{\left(1-2^{-m}\right) \zeta(m-1)(\nu t)^{m}}{m !}\right],
\end{aligned}
$$

where $\zeta(x)$ is Riemann's zeta function, and $\nu=1 / T=\omega_{0} / 2 \pi$

\section{The Green's function}

The Green's function $P(x, t)$ is defined as the equilibrium average

$$
P(x, t)=\langle\delta(x(t)-x(0)-x)\rangle,
$$

where $x(t)$ is the position of the moving particle at time $t$. For a particle moving on a fixed interval the calculation of (14) is trivial again and the result is

$$
\begin{aligned}
& \langle\delta(x(t)-x(0)-x)\rangle_{\tau}=\frac{1}{2}\left[\left(1-\frac{2 t}{\tau}\right)\right. \\
& \quad \times \sum_{n=-\infty}^{\infty}\{\delta(2 n L+|x|-v|t|)+\delta(2 n L-|x|-v|t|)\} \\
& \left.\quad+\frac{1}{L} \sum_{n=-\infty}^{\infty}\{\Theta[v|t|-(|x|+2 L)]-\Theta[v|t|-\{(2 n+1) L-|x|\}]\}\right]
\end{aligned}
$$

Application of the equilibrium average over $\tau$ with the distribution (3) yields

$$
\begin{aligned}
P(x, t)= & \frac{1}{2 L}\left[\mathrm{e}^{-|x| / L} \delta(|x|-v t)\right. \\
& +\sum_{n=1}^{\infty}\left\{\Theta(v|t|-(2 n+1)|x|) \frac{v|t|-(2 n+1)|x|-2 n L}{2 n L} \exp \left(-\frac{v|t|-|x|}{2 n L}\right)\right. \\
& \left.\left.+\Theta(v|t|-(2 n-1)|x|) \frac{v|t|-(2 n-1)|x|+2 n L}{2 n L} \exp \left(-\frac{v|t|+|x|}{2 n L}\right)\right\}\right] .
\end{aligned}
$$


Just as was done for the velocity autocorrelation function, eq. (15) may be expanded in a Fourier series. Here it takes the form

$$
\begin{aligned}
& \langle\delta(x(t)-x(0)-x)\rangle_{\tau}=\sum_{n=-\infty}^{\infty} \frac{1}{v \tau} \Theta(v \tau-2|x|) \\
& \quad \times\left\{\left(1-\frac{2|x|}{v \tau}\right) \cos \frac{2 \pi n x}{v \tau}-\frac{1}{\pi n} \sin \frac{2 \pi n|x|}{v \tau}\right\} \exp (2 \pi \mathrm{int} / \tau) .
\end{aligned}
$$

Unlike for the velocity autocorrelation function the average over $P_{\mathrm{eq}}$ is not easy to perform for the individual terms in the Fourier series.

The intermediate scattering function $F(k, t)$, the spatial Fourier transform of the Green's function, for a fixed interval length can be written in either of the forms

$$
\begin{aligned}
\left\langle\mathrm{e}^{-\mathrm{k}(x(t)-x(0))}\right\rangle_{\tau}= & \Theta\left(\frac{\tau}{2}-t\right)\left[\left(1-\frac{2 t}{\tau}\right) \cos (k v t)+\frac{2}{k v \tau} \sin (k v t)\right] \\
& +\Theta\left(t-\frac{\tau}{2}\right)\left[\left(\frac{2 t}{\tau}-1\right) \cos (k v(\tau-t))+\frac{2}{k v \tau} \sin (k v(\tau-t))\right], \quad 0 \leqslant t<\tau \quad(18) \\
= & \sum_{n=-\infty}^{\infty} \frac{8(k v \tau)^{2}}{\left\{(2 \pi n)^{2}-(k v \tau)^{2}\right\}^{2}}\left\{1-(-1)^{n} \cos \frac{k v \tau}{2}\right\} \exp (2 \pi \mathrm{int} / \tau) .
\end{aligned}
$$

For times outside $[0, \tau)(18 \mathrm{a})$ is defined through its periodicity The incoherent scattering function $S(k, \omega)$ is defined as the temporal and spatial Fourier transform of the Green's function. For fixed interval length it follows from (18b) as

$$
\begin{aligned}
& \left\langle\int_{-\infty}^{\infty} \mathrm{d} t \exp -1\{\omega t+k(x(t)-x(0))\}\right\rangle_{\tau} \\
& \quad=\sum_{n=-\infty}^{\infty} \frac{16 \pi(k v \tau)^{2}}{\left\{(2 \pi n)^{2}-(k v \tau)^{2}\right\}^{2}}\left(1-(-1)^{n} \cos \left(\frac{k v \tau}{2}\right)\right) \delta\left(\omega-\frac{2 \pi n}{\tau}\right)
\end{aligned}
$$

At this stage the average over $P_{\mathrm{eq}}(\tau)$ is easy to perform. We just give the result

$$
\begin{aligned}
S(k, \omega)= & \frac{2 \pi(k L)^{2}}{\omega\left\{\left(\pi \omega / \omega_{0}\right)^{2}-(k L)^{2}\right\}^{2}} \frac{\left.\left\{\cosh \left(\omega_{0} / \omega\right)+1\right\} \cos \left(k L \omega_{0} / \omega\right)+1\right\}}{\sinh \left(\omega_{0} / \omega_{0}\right)\left\{\cosh \left(\omega_{0} / \omega\right)+\cos \left(k L \omega_{0} / \omega\right)\right\}} \\
& +2 \pi \frac{\ln \left(1+(k L)^{2}\right)}{(k L)^{2}} \delta(\omega) .
\end{aligned}
$$

As a function of $k$ at fixed $\omega, S(k, \omega)$ exhibits a minimum at $k=0$, in marked contrast to normal diffusive behavior, where one has a Lorentzian line shape centered around $k=0$.

Furthermore, $S(k, \omega)$ is analytic in $k$, with simple poles at the positions 
$k=\left(2 \pi n \omega / \omega_{0} \pm i\right) / L$. Similarly, as a function of $\omega$ it has an essential singularity at $\omega=0$ and poles at $\omega=\omega_{0}(k L \pm \mathrm{i}) / 2 \pi n$. Finally notice that (20) satisfies the general relation $\lim _{k \rightarrow 0} k^{-2}(S(0, \omega)-S(k, \omega))=2 D(\omega) / \omega^{2}$.

\section{Different ensembles}

\section{Quenched period distribution}

In this case the equilibrium distribution of periods $P_{\text {eq }}$ is replaced by the "quenched distribution"

$$
P_{\mathrm{qu}}(\tau)=\frac{1}{T} \mathrm{e}^{-\tau / T}
$$

With this choice all averages may be performed just as well. We just give the form of the velocity autocorrelation function, analogous to (7), as

$$
\langle v(0) v(t)\rangle_{\mathrm{qu}}=\sum_{n=-\infty}^{\infty} \frac{8 v^{2}(1+\mathrm{i})(t / T)^{1 / 2}}{\{(2 n+1) \pi\}^{3 / 2}} K_{1}\left\{(1+\mathrm{i}) \sqrt{(2 n+1) 2 \omega_{0}}\right\}
$$

Hence the asymptotic long time behavior remains of order exp- $\left(2 \omega_{0}\right)^{1 / 2}$, although with a prefactor that increases by a factor $t^{-1 / 2}$ more slowly than in the equilibrium case. We will not bother to give the quenched averages of other functions here.

\subsection{Other period distributions}

Consider first general gamma distributions of the form

$$
P_{\mu}(\tau)=(T \Gamma(\mu))^{-1}\left(\frac{\tau}{T}\right)^{\mu-1} \mathrm{e}^{-\tau / T} .
$$

Under these distributions the velocity autocorrelation function remains of the same structure as in (7), but with $K_{2}$ replaced by $K_{\mu}$, with the same argument and with a prefactor proportional to $(t / \tau)^{\mu / 2}$.

Let us consider the more general case of an arbitrary function $f(t, \tau)$, that is periodic in $t$ with period $\tau$, and which is averaged over $P_{\mu}(\tau)$. Such a function may always be expanded in a Fourier series of the form

$$
f(t, \tau)=\sum_{n=-\infty}^{\infty} a_{n}(\tau) \mathrm{e}^{2 \pi n t / \tau} .
$$

A common occurrence is that $f(t, \tau)$ is a homogeneous function of order $\alpha$, 1.e it can be written as $f(t, \tau)=\tau^{\alpha} \varphi(t / \tau)$ In that case all the Fourier 
coefficients in (23) are of the form $a_{n}(\tau)=a_{n}(\tau / T)^{\alpha}$ with $a_{n}$ independent of $\tau$ Then the average over $P_{\mu}$ yields

$$
\int_{0}^{\infty} \mathrm{d} \tau P_{\mu}(\tau) f(t, \tau)=\frac{2}{\Gamma_{(\mu)}} \sum_{n=-\infty}^{\infty} a_{n}\left\{\frac{(2 n+1) 2 \pi \mathrm{i} t}{T}\right\}^{(\alpha+\mu) / 2} K_{\alpha+\mu}\left\{2 \sqrt{(2 n+1) \hat{\mathrm{i}} \omega_{0} t}\right\} .
$$

In the case of the velocity autocorrelation function the function $f(t, \tau)$ is homogeneous of degree zero The Green's function for fixed interval length is not a homogeneous function of $t$ and $\tau$, however, its spatial Fourier transform $F_{\tau}(k, t)$ is seen from (15) to be of the form $F_{\tau}(k, t)=f(k v t) \varphi(t / \tau)$. On the other hand one has the relation

$$
F(k, t)=\sum_{n=0}^{\infty} \frac{\left(-k^{2}\right)^{n}}{(2 n)^{1}}\left\langle(x(t)-x(0))^{2 n}\right\rangle .
$$

Hence one may conclude that the $2 n$th moment of displacement is a homogeneous function of degree $2 n$, which for large time differs from its limiting value by $t^{n+\mu / 2-1 / 4} \cos \left(\pi / 8+\left(2 \omega_{0} t\right)^{1 / 2}\right) \exp -\left(2 \omega_{0} t\right)^{1 / 2}$, as follows from (24) and (8)

Next, consider what happens for less smooth distributions. As an example consider a distribution that is uniform for $\tau_{0}<\tau<\tau_{1}$ and vanishes elsewhere Suppose the function to be averaged is homogeneous in $t$ and $\tau$, of order $\alpha$ Then the average of a typical Fourier component is of the form

$$
\begin{aligned}
\int_{\tau_{0}}^{\tau_{1}} \mathrm{~d} \tau \tau^{\alpha} \mathrm{e}^{2 \pi \ln t \tau}= & (2 \pi n t)^{\alpha+1} \int_{2 \pi n t / \tau_{1}}^{2 \pi n \tau / \tau_{0}} \mathrm{~d} u \mathrm{e}^{i u} u^{-(2+\alpha)} \\
= & (2 \pi n t)^{\alpha+1}\left\{\left.\frac{\mathrm{e}^{i u}}{1 u^{2+\alpha}}\right|_{2 \pi n t / \tau_{1}} ^{2 \pi n t / \tau_{0}}+\mathcal{O}\left(t^{-(3+\alpha)}\right)\right\} \\
& \sim \frac{1}{t} \times\left(\text { oscillating functions of periods } \frac{n}{\tau_{0}} \text { and } \frac{n}{\tau_{1}}\right)
\end{aligned}
$$

This result can easily be generalized suppose the distribution of perods is $n-1$ times differentiable but has a jump in its $n$th derivative at $\tau=\tau_{0}$ Then the long time behavior of the $n$th Fourier component of a periodic function with period $\tau$ contains a term of the type $t^{-(1+n)} \exp \left(2 \pi \mathrm{int} / \tau_{0}\right)$.

\subsection{Maxwellian distribution of velocittes}

Realistic models of statistical mechanics usually contain large numbers of moving particles, with speeds that are not all equal, but rather are dispersed 
according to some velocity distributıon. For classical systems in equilibrium this is just the Maxwellian distribution $(\beta m / 2 \pi)^{1 / 2} \exp \left(-\beta m v^{2} / 2\right)$ with $\beta=$ $1 / k_{\mathrm{B}} T, T$ is the temperature and $k_{\mathrm{B}}$ Boltzmann's constant To investigate the effects of velocity dispersion on our results we have to subject the averages that were calculated for a particle moving at constant speed, to a further average over the velocity distribution Application of this average to Grassberger's series (4) leads to a series of error functions, which is not very convenient again for extracting the long time behavior. Averaging of (7) with a Maxwellian distribution leads to complicated integrals, which do not seem to be well known, but the Fourier series (6) for fixed interval length can be averaged easily with the result

$$
\begin{aligned}
& \int_{-\infty}^{\infty} \mathrm{d} v\left(\frac{\beta m}{2 \pi}\right)^{1 / 2} \exp \left(-\beta m v^{2} / 2\right)\langle v(0) v(t)\rangle_{2 L / v l} \\
& \quad=\sum_{n=-\infty}^{\infty} \frac{4}{[(2 n+1) \pi]^{2} \beta m} \exp \left\{-\frac{[(2 n+1) \pi t]^{2}}{2 \beta m l^{2}}\right\}\left(1-\frac{[(2 n+1) \pi t]^{2}}{\beta m l^{2}}\right) .
\end{aligned}
$$

Notice that in this expression no oscillatory behavior is left. In order to obtain the final expression for the velocity autocorrelation function, one has to average (26) over the interval length distribution $P_{\text {eq }}(l)=\left(l / L^{2}\right) \exp (-l / L)$. To our knowledge the resulting integrals of type $\int_{0}^{x} \mathrm{~d} x x^{n} \exp -\left(\alpha x+\beta t^{2} / x^{2}\right)$ are not known in closed form, but for large $t$ they can be evaluated by the steepest descent method, as the exponent has a pronounced minimum at $x=\left(2 \beta t^{2} / \alpha\right)^{1 / 3}$ The dominant contribution to the long time behavior of the velocity autocorrelation function comes from the terms in (26) with $n=0,-1$. Its asymptotic form is

$$
\langle v(0) v(t)\rangle \approx-\left(\frac{\pi^{2} t^{2}}{\beta m L^{2}}\right)^{5 / 6}\left(\frac{2}{3 \pi^{3}}\right)^{1 / 2} \frac{8}{\beta m} \exp -\frac{3}{2}\left(\frac{\pi^{2} t^{2}}{\beta m L^{2}}\right)^{1 / 3} .
$$

Naively one might have expected a long time decay as $t^{-3}$, since after a time $t$ a large fraction of all particles with velocity $<L / t$ have maintained their initial velocity without colliding and their contribution to the velocity autocorrelation function readily follows to be proportional to $t^{-3}$ Apparently, however, these contributions are almost completely cancelled by those from particles that have completed just one collision, etcetera. Remarkably the remaining velocity autocorrelation function decays even faster than in the case of a single speed'

Application of the velocity average to the Green's function is possible in principle, but we have not worked this out. From the structure of (17) nevertheless, it appears that, except for the $n=0$-term describing the limiting 
long time behavior, all terms in the Fourier series, after averaging over $v$ and $l$, decay likewise as $\exp \left(-c t^{2 / 3}\right)$ for large $t$

\section{Conclusion}

We have shown that for the one-dimensional Lorentz gas, consisting of Poisson distributed fixed point scatterers and light particles moving at a given constant speed between two of such scatterers, the velocity autocorrelation function, decays asymptotically roughly as $\exp \left(-c t^{1 / 2}\right)$ for large time Superimposed on this behavior are oscillations with a period increasing as $t^{1 / 2}$ with time. This type of behavior manifests itself also in stochastic Lorentz models ${ }^{15}$ ) with not too small reflection rates. It seems plausible that a similar asymptotic decay will arise for higher dimensional Lorentz gases above the percolation density, but we expect that in that case the oscillations will be washed out since the motion of the light particles is not strictly periodic.

An asymptotic decay as $\exp \left(-c t^{1 / 2}\right)$ is rather unusual It is noteworthy in this context that this type of decay is also found for the velocity autocorrelation function of a particle moving diffusively in one dimension between two reflecting boundaries ${ }^{22}$ ). More generally, if a periodic function of period $\tau$ is averaged with a weight of type $\tau^{\alpha} \exp (-\tau / T)$, an asymptotic decay as $t^{\alpha / 2+1 / 4} \exp \left(-c t^{1 / 2}\right)$ results, with oscillations superimposed. However, an average over a weight function having a jump in the $n$th derivative at $\tau=\tau_{0}$ gives rise to slowly decaying oscillations of type $t^{-(n+1)} \exp \left(2 \pi \mathrm{imt} / \tau_{0}\right)$ with $m$ an integer

Finally, an average over velocities with a Maxwellian distribution reduces the asymptotic behavior of the velocity autocorrelation function to $t^{5 / 3} \exp (-$ $\left.c^{\prime} t^{2 / 3}\right)$ with $c^{\prime}$ some constant again. In addition it suppresses the oscillations completely

\section{Acknowledgements}

This work was supported, in part, by NSF Grant CHE-8009555 and the Heinrich Hertz Stiftung RMM would like to thank Prof Dr. F. Schlögl for his hospitality at the RWTH.

\section{Appendix}

To prove that the velocity autocorrelation function decays faster than any power of $t$ we need the following theorem: 
Theorem. Let the function $\hat{f}$ be $C^{\infty}$ on $(-\infty, \infty)$, let the inverse Fourier transform $f$ of $\hat{f}$ exist and let

$$
\frac{\mathrm{d}^{n} \hat{f}(\omega)}{\mathrm{d} \omega^{n}}=\mathcal{O}\left(|\omega|^{-(1+\epsilon)}\right), \quad \text { as }|\omega| \rightarrow \infty, n=1,2 \quad \ldots
$$

Then $f$ satisfies $t^{n} f(t)<C_{n}, n=1,2$. .

Proof One has

$$
t^{n} f(t)=\int_{-\infty}^{\infty} \mathrm{d} \omega \mathrm{e}^{i \omega t}\left(\mathrm{i} \frac{\mathrm{d}}{\mathrm{d} \omega}\right)^{n} \hat{f}(\omega)
$$

by repeated partial integration Hence

$$
\left|t^{n} f(t)\right| \leqslant \int_{-a_{n}}^{a_{n}} \mathrm{~d} \omega\left|\frac{\mathrm{d}^{n} \hat{f}(\omega)}{\mathrm{d} \omega^{n}}\right|+2 b_{n} \int_{a_{n}}^{\infty} \mathrm{d} \omega \omega^{-(1+\epsilon)} \equiv C_{n},
$$

where $a_{n}, b_{n}$ and $C_{n}$ are positive constants depending on $n$.

The expression for $D(\omega)$ given in (12) satisfies the requirements of the theorem. It has an essential singularity at the origin, yet is infinitely differentrable on the real axis At $\pm \infty$ the $n$th derivative decays as $\omega^{-(n+2)}$. Hence it follows that the velocity autocorrelation function decays faster than any power of $t$ for $t \rightarrow \pm \infty$

\section{References}

1) See e g Donald A McQuarrıc, Statıstıcal Mechanıcs (Harper \& Row, New York 1976) p $512 \mathrm{ff}$

2) $Y$ Pomeau and P Résiboss, Phys Reports C19 (1975) 63, and references quoted there

3) T E Wainwright, B J Alder and D M Gass, Phys Rev A4 (1971) 233

4) K Kawasakı, Phys Lett A34 (1971) 12

5) H A Lorentz, Proc Roy Acad Amst 7 (1905) 438, 585, 684

6) E H Hauge, in Transport Phenomena, G Kirczenow and J Marro eds, Lecture notes in Physics vol 31 (Springer, Berlın, 1974) p 337

7) M H Ernst and A Weyland, Phys Lett A34 (1971) 39

8) C Bruın, Physica 72 (1974) 261

9) J C Lewis and J A Tjon, Phys Lett A 66 (1978) 349

10) B J Alder and W E Alley, J Stat Phys 19 (1978) 341

B J Alder, in Stochastic Processes in Nonequilibrium Systems, L Garrido, P Seglar and P J Shepherd eds, Lecture Notes in Physıcs vol 84 (Sprınger, Berlın, 1978) p 168

W E Alley, Ph D thesis, Univ Calıfornia, Davis (1979)

11) J R Dorfman, in Fundamental Problems in Statıstical Mechanics vol 3, E G D Cohen, ed (North-Holland, Amsterdam, 1975) p 289

12) V K S Shante and S Kirkpatrick, Adv Phys 20 (1973) 325 
13) S W Haan and R Zwanzig, J Phys A10 (1977) 1547

14) $T$ Vicsek and J Kertész, J Phys A14 (1981) L31

15) P Grassberger, Physica 103A (1980) 558

16) Henk van Beijeren, Rev Mod Phys 54 (1982) 195

Henk van Beljeren and Herbert Spohn, J Stat Phys to appear

17) J J Erpenbeck, unpublished

18) $R$ Dumcke, unpublished

19) I S Gradshteyn and I M Ryzkık, Table of Integrals Series and Products (A P , New York, 1965) eq 34719

20) I S Gradshteyn and I M Ryzhık, Tables of Integrals Serıes and Products (A P, New York, 1965) eq 84516

21) Niels Nielsen, Die Gammafunktion (Chelsea, New York, 1965) p 16

22) Christian Hemsing, unpublished 